\title{
Optimal Detection Ordering for Coded V-BLAST
}

\author{
Alain U. Toboso, Sergey Loyka, and Francois Gagnon
}

\begin{abstract}
Optimum ordering strategies for the coded Vertical Bell Labs Layered Space-Time (V-BLAST) architecture with capacity achieving temporal codes on each stream are analytically studied, including 4 different power/rate allocation strategies among data streams. Compact closed-form solutions are obtained for the case of zero-forcing (ZF) V-BLAST with two transmit antennas and necessary optimality conditions are found for the general case. The optimal rate allocation is shown to have a major impact (stronger streams are detected last) while the optimal power allocation does not alter the original Foschini ordering (stronger streams are detected first). Sufficient conditions for the optimality of the greedy ordering are established: it is optimal for the ZF V-BLAST under an optimal rate allocation with two transmit antennas at any SNR and with any number of antennas in the low and high SNR regimes. It satisfies the necessary optimality conditions for larger systems at any SNR and is nearlyoptimal in many cases. An SNR gain of ordering is introduced and studied, including closed-form expressions as well as lower and upper bounds and the conditions for their achievability. For the minimum mean square error (MMSE) V-BLAST under an optimal rate allocation, any ordering is shown to deliver the same system capacity. All the results also apply to a multiple-access channel with the successive interference cancelation receiver.
\end{abstract}

Index Terms-MIMO, V-BLAST, optimal ordering, successive interference cancellation.

\section{INTRODUCTION}

$\mathbf{T}$ HE multiple-input multiple-output (MIMO) communication architecture has been widely adopted by the academia and industry due to its high spectral efficiency unattainable by conventional techniques [1]. To reduce its processing complexity, Vertical Bell Labs Layered Space-Time (V-BLAST) was proposed in [2][3] as a low-complexity architecture that is able to achieve a substantial portion of the total MIMO channel capacity given that the multipath environment is rich enough and capacity-approaching temporal codes (e.g. LDPC, turbo or polar codes) are used for each data stream [4][21]. In addition to spatial multiplexing at the transmitter, its key processing steps at the receiver are (i) interference cancellation from already detected symbols (i.e. successive interference cancellation (SIC)), (ii) interference nulling from yet-to-be-detected symbols (either zero-forcing or MMSE), and (iii) an optimal detection ordering procedure to optimize the overall performance.

While unordered V-BLAST analysis is feasible [5][7][8], the optimal ordering procedure presents a significant problem

Manuscript received April 10, 2013; revised September 10 and November 15,2013 . The editor coordinating the review of this paper and approving it for publication was M. Matthaiou.

This paper was presented in part at 13th Canadian Workshop on Information Theory (CWIT'13), Toronto, Canada, June 2013.

A. U. Toboso and S. Loyka are with the School of Electrical Engineering and Computer Science, University of Ottawa, Ontario, Canada (e-mail: sergey.loyka@ieee.org).

F. Gagnon is with the Department of Electrical Engineering, Ecole de Technologie Suprieure, Montreal, Canada (e-mail: francois.gagnon@etsmtl.ca).

Digital Object Identifier 10.1109/TCOMM.2013.121413.130268 for the analysis so that only the two Tx antennas case has been fully settled [6]. A number of approximate results (highSNR) or bounds have been reported in [9][10] for the general case. Due to these difficulties and also because its smaller complexity, unordered V-BLAST became popular [5][7][8]. Since its performance may be not satisfactory in some cases, various optimization techniques have been proposed, e.g. optimal power and/or rate allocation among data streams, nearML sphere decoding etc. [12]-[19], which can be considered as an alternative to a computationally-demanding optimal ordering procedure. Optimal power and/or rate allocations for the unordered, uncoded and coded ZF V-BLAST have been obtained in [14] and [18][19] and their performance have also been analyzed, demonstrating significant benefit of such optimization.

The optimal ordering procedure requires $m$ ! orderings to be compared in the general case, where $m$ is the number of Tx antennas. This can be prohibitively complex for large $m$ in realtime implementations. Thus, various sub-optimal orderings have been proposed [9][16][17]. The greedy ordering, which is based on the "strongest-goes-last" principle, was introduced in an ad-hoc way in [17] and its advantage was demonstrated via simulations. It was further shown in [16] to achieve the optimal diversity-multiplexing tradeoff (DMT), but this holds only asymptotically $(S N R \rightarrow \infty)$ and in i.i.d. Rayleigh-fading channel. It is not clear what the finite-SNR implications are ${ }^{1}$ and whether this holds for other fading distributions or for a fixed (static) channel. The present paper will answer these questions.

An optimal ordering for the V-BLAST is a hard geometric combinatorial (and hence non-convex [22]) problem in general. While Foschini et al [2][3] has found the optimal ordering to minimize the overall block error rate for a given (fixed) channel and the essentially uncoded system under uniform power/rate allocation, in which stronger streams are detected first, no analytical solution is known to date for a coded V-BLAST with an optimal power/rate allocation. The only remaining option explored in the literature is a brute force approach by comparing all $m$ ! orderings numerically.

In the present paper, we study optimal ordering for coded V-BLAST under 4 different power/rate allocation policies, provide closed-from analytical solutions for the $m=2$ case and compare the performance improvement they bring in. The considered power/rate allocation policies are (i) the uniform power/rate allocation (UPRA); (ii) an optimal instantaneous (for a given fixed channel) rate allocation (IRA); (iii) an optimal instantaneous power allocation (IPA); (iv) an optimal instantaneous power/rate allocation (IPRA) ${ }^{2}$. In the case of the

\footnotetext{
${ }^{1}$ since better DMT does not imply better finite-SNR performance, even at high SNR, see e.g. [24][25].

${ }^{2}$ see [19] for details of these allocation policies. They are motivated by modern adaptive systems that make use of variable-power amplifiers and/or variable modulation/coding, as in 3 or $4 \mathrm{G}$ systems [20].
} 
UPRA or the IPA (i.e. when the rate allocation is uniform), an optimal ordering is the original Foschini ordering, i.e. stronger streams are detected first [2]-[4]. In the case of the IRA or IPRA, i.e. under an optimal rate allocation, an optimal ordering is just the opposite: stronger stream are detected last, thus revealing a dramatic impact of the rate allocation policy on an optimal ordering procedure.

In the general case of $m>2 \mathrm{ZF}$ V-BLAST, we provide compact necessary optimality conditions, which depend on the channel matrix only and are independent of the SNR and other system parameters. These conditions can be used to rule out many of the possible $m$ ! combinations so that the bruteforce approach can be applied to a much-smaller set and thus becomes practically-feasible. These conditions also provide a number of insights into the optimal ordering procedure and its properties which cannot be obtained numerically. In particular, their reveal essentially the same dramatic impact of the rate allocation policy on an optimal ordering as in the $m=2$ case.

The sufficient optimality conditions of Section III show that the greedy ordering of [16][17] is actually optimal one under the IRA/IPRA for the $m=2$ case and in any fixed channel or, equivalently, for any realization of a slowly-fading channel, i.e. "point-wise". This point-wise optimality implies statistical optimality (in terms of outage or ergodic capacity or outage probability) for any fading distribution. In the $m>2$ case, the greedy ordering is shown to meet the necessary optimality conditions under the IRA/IPRA and is nearly optimal in many cases. We attribute this to the fact that the greedy ordering algorithm, while not finding a best ordering in general, does eliminate most "bad" orderings.

To quantify the impact of optimal ordering, an SNR gain of ordering is introduced and studied, including compact analytical solutions and upper/lower bounds and conditions for their achievability. The coded MMSE V-BLAST under the IRA/IPRA is shown to have the same system capacity under any ordering and thus an optimal detection ordering procedure is not required, which provides an extra incentive (less complexity) to use MMSE rather than ZF V-BLAST. This is in stark contrast to the MMSE V-BLAST under the UPRA, where optimal ordering improves the performance significantly [10].

The major insight from this study is that the optimal rate allocation among data streams has a much more pronounced impact on the optimal ordering (stronger streams are detected last) as opposed to the optimal power allocation, which does not alter the original Foschini ordering (stronger streams are detected first), regardless of whether temporal coding is used or not.

Finally, we mention that the V-BLAST system architecture naturally represents the multiple-access channel (MAC), i.e. an unlink of a cellular system, under successive interference cancellation so that all our results, including optimal user detection order, also apply to such setting.

The rest of the paper is organized as follows. Section II introduces the basic system and channel model. In Section III, we consider the ZF V-BLAST and find sufficient and necessary ordering optimality conditions under four different power/rate allocation policies. The SNR gain of ordering is introduced and studied in Section IV. The greedy ordering is considered in Section V and its optimality is shown for the $m=2$ case. Section VI deals with the MMSE V-BLAST. Finally, Section VII concludes the paper.

\section{SYSTEM MODEL}

The standard discrete-time MIMO channel model is

$$
\mathbf{r}=\mathbf{H} \Lambda \mathbf{q}+\boldsymbol{\xi}=\sum_{i=1}^{m} \mathbf{h}_{i} \sqrt{\alpha_{i}} q_{i}+\boldsymbol{\xi}
$$

where $\mathbf{q}=\left[q_{1}, q_{2}, \ldots, q_{m}\right]^{T}$ and $\mathbf{r}=\left[r_{1}, r_{2}, \ldots, r_{n}\right]^{T}$ are the transmitted and received signal vectors respectively, $\mathbf{H}=$ $\left[\mathbf{h}_{1} . . \mathbf{h}_{m}\right]$ is the $n \times m$ channel matrix ( $n \mathbf{R x}$ and $m \mathrm{Tx}$ antennas, $n \geq m$ ) representing the complex channel gains from each transmit to each receive antenna, and $\mathbf{h}_{i}$ is its $\mathbf{i}$-th column. The channel matrix $\mathbf{H}$ is assumed to be fixed (e.g. a given realization of a quasi-static fading channel) so that the standard infinite-horizon information theory assumption holds; $\boldsymbol{\xi}$ is the circularly symmetric additive white Gaussian noise vector with i.i.d. entries i.e. $\boldsymbol{\xi} \sim C N\left(0, \sigma_{0}^{2} \mathbf{I}\right)$. $\Lambda$ is a diagonal matrix whose entries are $\sqrt{\alpha_{i}}$, where $\alpha_{i}$ represents the normalized power allocation to i-th stream, $\sum_{i} \alpha_{i}=m$. We assume that the receiver has full channel state information (CSI), while the transmitter has a partial CSI in the form of powers and rates allocated to various streams.

The V-BLAST detection algorithm includes 3 major steps ${ }^{3}$ :

(i) interference cancelation from already detected symbols (the SIC);

(ii) interference nulling (ZF or MMSE) from yet-to-bedetected symbols via orthogonal projections; unless otherwise indicated, we will assume ZF interference nulling;

(iii) an optimal detection ordering to improve overall system performance.

After the interference cancellation and ZF nulling steps and for the standard ordering (i.e. stream 1 is detected first etc.), which is also known as unordered detection, the equivalent scalar channel of the $i$-th stream is [18][19],

$$
r_{i}=\left|\mathbf{h}_{i \perp}\right| \sqrt{\alpha_{i}} q_{i}+\widetilde{\xi}_{i},
$$

where $r_{i}$ is the $i$-th component of $\mathbf{r}$ after the projection, $\mathbf{h}_{i \perp}$ is the projection of $\mathbf{h}_{i}$ onto the sub-space orthogonal to that spanned by yet-to-be-detected streams, i.e. $\mathbf{h}_{i \perp} \perp\left\{\mathbf{h}_{i+1}, \ldots, \mathbf{h}_{m}\right\},|\mathbf{h}|$ is the Euclidean norm (length) of vector $\mathbf{h}$, and $\widetilde{\xi}_{i}$ is the projected noise of $i$-th stream (still Gaussian after the projection). Our analysis below is based on information-theoretic principles as in e.g. [20][21]. Assuming that each stream employs a capacity-achieving temporal code ${ }^{4}$, this stream can support a target rate $R_{i}$ up to its instantaneous capacity ${ }^{5}$ given by

$$
C_{i}=\ln \left(1+\left|\mathbf{h}_{i \perp}\right|^{2} \alpha_{i} \gamma\right)[\mathrm{nat} / \mathrm{s} / \mathrm{Hz}],
$$

where $\gamma=1 / \sigma_{0}^{2}$ is the average SNR at each Rx antenna, with arbitrary-low probability of error, which can be reduced to any desired value by using sufficiently long codewords [20][21]. As a consequence, the use of capacity-achieving codes at each

\footnotetext{
${ }^{3}$ See e.g. [2]-[4][8][9][21] for further details of these steps and their mathematical models.

${ }^{4}$ this models well practical codes operating very close to the capacity, e.g. LDPC, turbo or polar codes [20][21].

5 i.e. the capacity for a given (fixed) channel realization.
} 
stream under the condition $R_{i} \leq C_{i}$ eliminates the error propagation effect, which is in stark contrast to the uncoded V-BLAST, where error propagation degrades the performance significantly [5]-[10]. Unlike the uncoded system, the weakest stream does not necessarily dominate the performance of coded V-BLAST.

The total system capacity $C$, which includes the channel as well as the transmission and reception strategy, depends on the power and rate allocation strategy [18][19]. When the uniform rate/power allocation (UPRA) is used, i.e. all streams transmit at the same target rate and using the same power $\left(\alpha_{i}=1\right)$, the system capacity is limited by the weakest stream so that

$$
C_{U P R A}=m \min _{i} C_{i}=m \ln \left(1+\min _{i}\left|\mathbf{h}_{i \perp}\right|^{2} \gamma\right) .
$$

When the optimal instantaneous rate allocation (IRA) is used, i.e. the rate of each stream is adjusted to match its capacity, $R_{i}=C_{i}$, under the uniform power allocation, the system capacity is

$$
C_{I R A}=\sum_{i=1}^{m} C_{i} .
$$

This two strategies can be further combined with the instantaneous power allocation to maximize the system capacity [19]. Comparing (4) to (5), we conclude that while the weakest stream does dominate the system performance under the UPRA, it is not the case for the IRA.

We note that this system model also applies to a multipleaccess channel (MAC) where different streams represent difference users, e.g. an uplink of a cellular system, so that all our results will also hold in that scenario as well.

To further improve the system performance, the stream detection order can be optimized to maximize the system capacity [16][17]. Let $\pi=\left\{k_{1}, k_{2}, \ldots, k_{m}\right\}$ represents the detection order where stream $k_{1}$ is detected first etc. All the capacities above then become the functions of the detection order. Changing the detection order is equivalent to swapping the columns of the channel matrix $\mathbf{H}$ so that the re-ordered matrix $\mathbf{H}_{\pi}=\left[\mathbf{h}_{k_{1}} . . \mathbf{h}_{k_{m}}\right]$ represent detection ordering $\pi$.

Below, we consider an optimal ordering strategy for each of the power/rate allocation strategies. It turns out that it is the rate allocation strategy that affects the optimal detection ordering most. To make the analysis tractable, we consider first the case of $2 \mathrm{Tx}$ antennas, and generalize the results later to the $m>2$ case.

\section{OPTIMUM ORDERING FOR ZF V-BLAST}

In this section, we consider four instantaneous power/rate allocation strategies (uniform/optimal for power/rate) for the ZF V-BLAST to see the impact they have on optimal ordering. By comparing them, we observe that it is the IRA that brings the largest incremental improvement; using the optimal power allocation on top of it brings little improvement. For the case of $m=2$, we give explicit closed-form solutions, while for $m>2$, we provide necessary optimality conditions.

\section{A. Optimum Ordering Under the IRA}

Under the IRA, the per-stream rates are adjusted to match the per-stream capacities with the uniform power allocation.
The optimum detection ordering maximizes the instantaneous sum capacity of the system,

$$
\pi^{*}=\arg \max _{\pi} C_{I R A}(\pi)=\arg \max _{\pi} \sum_{i=1}^{m} C_{i}(\pi),
$$

where $C(\pi)$ and $C_{i}(\pi)=\ln \left(1+\left|\mathbf{h}_{k_{i} \perp}\right|^{2} \gamma\right)$ are the total system capacity and the per-stream capacity as functions of the detection ordering $\pi$, and $\mathbf{h}_{k_{i} \perp}$ is the projection of $\mathbf{h}_{k_{i}}$ orthogonal to $\left\{\mathbf{h}_{k_{i+1}} . . \mathbf{h}_{k_{m}}\right\}$.

For $m=2$, the optimum detection order is as follows.

Proposition 1: The optimum detection ordering for the coded V-BLAST with two Tx and $n \mathrm{Rx}$ antennas under the IRA is to detect the strongest stream (with highest unprojected channel gain) last,

$$
\pi^{*}=\arg \max _{\pi} \sum_{i=1}^{2} C_{i}(\pi)=\{1,2\} \text { iff }\left|\mathbf{h}_{1}\right| \leq\left|\mathbf{h}_{2}\right| .
$$

The "only if" part in (7) holds when $\mathbf{h}_{1}, \mathbf{h}_{2}$ are not orthogonal, $\phi \neq \pi / 2$, where $\phi$ is the angle between them. When $\phi=\pi / 2$ and/or $\left|\mathbf{h}_{1}\right|=\left|\mathbf{h}_{2}\right|$, any ordering delivers the same system capacity.

Proof: Let $g_{i}=\left|\mathbf{h}_{i}\right|^{2}, \beta=\sin ^{2} \phi, \pi_{1}=\{1,2\}, \pi_{2}=$ $\{2,1\}$. If $\beta=1$, any ordering is optimal (since the streams are independent), so that the assertion holds trivially. Thus, assume $\beta<1$ and $C\left(\pi_{1}\right) \geq C\left(\pi_{2}\right)$, and observe that the following chain of inequalities hold:

$$
\begin{aligned}
C\left(\pi_{1}\right)-C\left(\pi_{2}\right) & \geq 0 \\
\Rightarrow\left(1+\gamma \beta g_{1}\right)\left(1+\gamma g_{2}\right)-\left(1+\gamma \beta g_{2}\right)\left(1+\gamma g_{1}\right) & \geq 0 \\
\Rightarrow(1-\beta) g_{2}-(1-\beta) g_{1} & \geq 0 \\
\Rightarrow g_{2} & \geq g_{1},
\end{aligned}
$$

which proves the "only if" part. The "if" part can be proved by observing that the same chain of inequalities holds in the other direction.

Note that this ordering is opposite of that of the uncoded, unoptimized (the UPRA) V-BLAST [2][3][6], which detects the strongest stream first. It is also SNR and other system parameters-independent, since it is based on the channel matrix only. Unfortunately, as numerical observations indicate, this independence does not hold anymore for larger systems $(m>2)$, where, in general, an optimal ordering is SNRdependent.

However, using the same reasoning as in Proposition 1, a necessary optimality condition can be formulated for any $m$.

Proposition 2: Given that $\mathbf{h}_{k_{i-1} \perp}$ and $\mathbf{h}_{k_{i} \perp}$ are nonorthogonal to each other, an optimum channel ordering $\pi^{*}=$ $\left\{k_{1}, k_{2}, \ldots, k_{m}\right\}$ must satisfy the following necessary conditions:

$$
\left|\mathbf{h}_{k_{i-1} \perp}\right| \leq\left|\mathbf{h}_{k_{i} \perp}\right| \forall 2 \leq i \leq m,
$$

where $\mathbf{h}_{k_{i-1} \perp}$ and $\mathbf{h}_{k_{i} \perp}$ are the projections of vectors $\mathbf{h}_{k_{i-1}}$ and $\mathbf{h}_{k_{i}}$ orthogonal to the sub-space spanned by $\left\{\mathbf{h}_{k_{i+1}}, \ldots \mathbf{h}_{k_{m}}\right\}$. If some $\mathbf{h}_{k_{i-1} \perp}$ and $\mathbf{h}_{k_{i} \perp}$ are of equal length and/or orthogonal to each other, any ordering among them is optimum.

Proof: Consider two orderings $\pi_{1}=$ $\left\{k_{1}, . . k_{i-1}, k_{i}, \ldots k_{m}\right\}$ and $\pi_{2}=\left\{k_{1}, . . k_{i}, k_{i-1}, \ldots k_{m}\right\}$ (i.e. streams $k_{i}$ and $k_{i-1}$ are swapped over) and observe that the difference in their capacities is due to the contributions 
TABLE I

NUMBER OF ORDERINGS SATISFYING THE NECESSARY OPTIMALITY CONDITIONS.

\begin{tabular}{|l|l|l|l|l|l|}
\hline \multirow{2}{*}{$m$} & \multirow{2}{*}{ Total } & \multicolumn{4}{|c|}{ Remaining } \\
\cline { 3 - 6 } & & LB & $\%$ & UB & $\%$ \\
\hline 3 & 6 & 2 & 33.3 & 3 & 50 \\
\hline 4 & 24 & 5 & 20.8 & 6 & 25 \\
\hline 5 & 120 & 16 & 13.3 & 30 & 25 \\
\hline 6 & 720 & 61 & 8.5 & 90 & 12.5 \\
\hline 7 & 7040 & 272 & 5.4 & 880 & 12.5 \\
\hline 8 & 40320 & 1385 & 3.4 & 2520 & 6.3 \\
\hline
\end{tabular}

of the streams $k_{i}$ and $k_{i-1}$ only, since their swapping does not affect the capacity of the other streams ${ }^{6}$. Apply now Proposition 1 to the sum capacity of these two streams to obtain the desired result. We observe that the necessary conditions do not determine the optimal ordering uniquely in the general case but rather specify "suspicious" orderings which include an optimal one, i.e. they are not sufficient for optimality, and that optimal ordering is not unique in general ${ }^{7}$. In the general case, optimal ordering is SNR-dependent so that any ordering procedure that is based on the channel matrix only cannot provide optimal result in general.

While there may be more than one ordering satisfying these conditions for $m>2$, there is only one such ordering for the $m=2$ case, so that they are both sufficient and necessary as Proposition 1 indicates.

Three important properties follow from the necessary optimality conditions:

1) Given that all $\mathbf{h}_{k_{i} \perp}$ are of different length and nonorthogonal to each other, swapping two consecutive columns for a given order that meets the necessary optimality conditions results in a lower system capacity.

2) A (before-projection) weakest stream is never detected last.

3) The strongest (before-projection) stream is never detected 2nd last.

These properties allow one to reduce significantly the number of possible orderings during a brute-force combinatorial optimization, as Table I demonstrates by comparing the total number of orderings with lower (LB) and upper (UB) bounds to the number of remaining orderings that satisfy the necessary optimality conditions ${ }^{8}$. Clearly, the larger the system size, the larger the benefit offered by the necessary optimality conditions, which rule out most of the orderings thus reducing significantly the computational complexity of the ordering procedure.

\section{B. Optimum Ordering Under the UPRA}

The coded V-BLAST with uniform power and rate allocation (UPRA) among the data streams may be used to simplify the system design. Since its system capacity is dominated

\footnotetext{
${ }^{6}$ the streams detected before them are projected orthogonally to these two (interference nulling), and the later streams are not aware about their existence at all (interference cancelation).

${ }^{7}$ this can be seen by considering an orthogonal channel, where all column vectors are orthogonal to each other, for which any ordering is optimal.

${ }^{8}$ we use the bounds since an exact number is channel-dependent and finding it is a hard combinatorial problem for which a solution is not known.
}

by the weakest stream [19], $C=m \min _{i} C_{i}$, the optimum ordering is

$$
\pi^{*}=\arg \max _{\pi} \min _{i} C_{i}(\pi)=\arg \max _{\pi} \min _{i}\left|\mathbf{h}_{k_{i} \perp}\right|,
$$

where $\mathbf{h}_{k_{i} \perp}$ is orthogonal to $\left\{\mathbf{h}_{k_{i+1}}, \ldots \mathbf{h}_{k_{m}}\right\}$, i.e. maximizes the weakest after-projection stream gain.

In the case of $m=2$, this can be evaluated explicitly.

Proposition 3: The optimum detection ordering for the coded V-BLAST with two Tx and $n \mathrm{Rx}$ antennas under the UPRA is to detect the strongest (before-projection) stream first,

$$
\begin{aligned}
\pi^{*} & =\arg \max _{\pi} \min _{i} C_{i}(\pi) \\
& =\{1,2\} \text { iff }\left|\mathbf{h}_{1}\right| \geq\left|\mathbf{h}_{2}\right| \text { for } \forall \phi \neq 0 .
\end{aligned}
$$

The "only if" part in (10) holds if $\mathbf{h}_{1}$ and $\mathbf{h}_{2}$ are not orthogonal, $\phi \neq \pi / 2$. If $\phi=\pi / 2$ and/or $\left|\mathbf{h}_{1}\right|=\left|\mathbf{h}_{2}\right|$, any ordering delivers the same capacity. If $\phi=0$, the system capacity is zero for any ordering.

Proof: The proof is by contradiction. Assume that $\pi^{*}=$ $\{1,2\}$ is optimal but $g_{1}<g_{2}$ (and also $0<\beta<1$, otherwise any ordering is optimal). Therefore, for the other ordering $\pi=\{2,1\}$,

$$
\begin{gathered}
C_{1}(\pi)=\ln \left(1+\gamma \beta g_{2}\right)>C_{1}\left(\pi^{*}\right)=\ln \left(1+\gamma \beta g_{1}\right) \\
C_{2}(\pi)=\ln \left(1+\gamma g_{1}\right)>C_{1}\left(\pi^{*}\right)=\ln \left(1+\gamma \beta g_{1}\right)
\end{gathered}
$$

so that

$$
C(\pi)=\min _{i} C_{i}(\pi)>C_{1}\left(\pi^{*}\right) \geq \min _{i} C_{i}\left(\pi^{*}\right),
$$

a contradiction. Therefore, $g_{1} \geq g_{2}$.

Note that this is in fact the Foschini ordering. Hence, unlike the IRA, the power allocation strategy has no impact on the optimal ordering, even when coding is used.

This result can be further generalized to any $m$.

Proposition 4: Let $C_{i}=\min _{j} C_{j}$ be the smallest perstream capacity in the coded V-BLAST under the UPRA with any number of $\mathrm{Tx}$ and $\mathrm{Rx}$ antennas and let $C_{i}<C_{j} \forall j \neq i$. Then, a detection ordering $\pi^{*}=\left\{k_{1}, . ., k_{i}, k_{i+1}, . . k_{m}\right\}$ is optimum only if

$$
\left|\mathbf{h}_{k_{i} \perp}\right| \geq\left|\mathbf{h}_{k_{i+1} \perp}\right|,
$$

when $\mathbf{h}_{k_{i} \perp}$ and $\mathbf{h}_{k_{i+1} \perp}$ are non-orthogonal to each other (both are orthogonal to $\left\{\mathbf{h}_{k_{i+2}}, \ldots \mathbf{h}_{k_{m}}\right\}$ ), and any ordering among them offers the same capacity otherwise. Ordering of all the other streams is arbitrary as long as $i$-th stream remains the weakest one.

Proof: The proof is similar to that of Proposition 2. Observe that swapping streams $i$ and $i+1$ does not affect any other stream. Since $C_{i}=\min _{j} C_{j}$, if $\left|\mathbf{h}_{k_{i} \perp}\right| \geq\left|\mathbf{h}_{k_{i+1} \perp}\right|$ does not hold, one can increase the capacity of $i$-th stream by swapping these two streams (and keeping the rest of the ordering) due to Proposition 3 (we assume here that $\mathbf{h}_{k_{i} \perp}$ and $\mathbf{h}_{k_{i+1} \perp}$ are not orthogonal, otherwise any ordering among them offers the same capacity). This, in turn, will increase the system capacity, which is impossible for an optimal ordering, so that $\left|\mathbf{h}_{k_{i} \perp}\right| \geq\left|\mathbf{h}_{k_{i+1} \perp}\right|$ must hold. We note that the ordering of all other streams can be arbitrary as long as $C_{i}$ remains the smallest per-stream capacity, since it is the least-capacity stream that dominates the system performance. 
Thus, pairwise Foschini ordering around the least-capacity stream is necessary for optimality.

\section{Optimum ordering under the IPA}

Let us now consider the optimal instantaneous power allocation (IPA) under the uniform rate allocation, when different streams make use of the same code/modulation format. From [19], the system capacity under the IPA for a given ordering $\pi$ is given by

$$
C_{I P A}(\pi)=m \ln (1+\bar{g}(\pi) \gamma) \text { if }\left|\mathbf{h}_{k_{i} \perp}\right|>0 \forall i,
$$

and 0 otherwise, where $\bar{g}(\pi)$ is the harmonic mean per-stream power gain for a given ordering $\pi$,

$$
\bar{g}(\pi)=\left(\frac{1}{m} \sum_{i=1}^{m}\left|\mathbf{h}_{k_{i} \perp}\right|^{-2}\right)^{-1},
$$

where $\mathbf{h}_{k_{i} \perp}$ is orthogonal to $\left\{\mathbf{h}_{k_{i+1}}, \ldots \mathbf{h}_{k_{m}}\right\}$, so that the optimum ordering is to maximize the harmonic mean gain,

$$
\pi^{*}=\arg \max _{\pi} \bar{g}(\pi) .
$$

Note that this holds for any $m$ and is SNR-independent, as opposed to the case of the IRA. For $m=2$, one obtains:

Proposition 5: The optimum detection ordering for the coded V-BLAST with two Tx and $n \mathrm{Rx}$ antennas under the IPA (and uniform rate allocation) is to detect the strongest stream first (i.e. the Foschini ordering),

$$
\pi^{*}=\arg \max _{\pi} \bar{g}(\pi)=\{1,2\} \text { iff }\left|\mathbf{h}_{1}\right| \geq\left|\mathbf{h}_{2}\right| \forall \phi \neq 0
$$

The "only if" part in (15) holds when $\mathbf{h}_{1}$ and $\mathbf{h}_{2}$ are not orthogonal, $\phi \neq \pi / 2$. If $\phi=\pi / 2$ and/or $\left|\mathbf{h}_{1}\right|=\left|\mathbf{h}_{2}\right|$, any ordering delivers the same capacity.

Proof: First, observe that $C=0$ if $\phi=0$ and that any ordering is optimum if $\phi=\pi / 2$, so there is nothing to prove in these cases. Assume further that $0<\phi<\pi / 2$ and $C\left(\pi^{*}\right) \geq$ $C(\pi)$, where $\pi=\{2,1\}$, and note the following chain of inequalities:

$$
\begin{array}{r}
C\left(\pi^{*}\right) \geq C(\pi) \\
\Rightarrow \bar{g}\left(\pi^{*}\right) \geq \bar{g}(\pi) \\
\Rightarrow \frac{g_{2}+g_{1} \beta}{g_{1} g_{2} \beta} \leq \frac{g_{1}+g_{2} \beta}{g_{1} g_{2} \beta} \\
\Rightarrow g_{2}(1-\beta) \leq g_{1}(1-\beta) \\
\Rightarrow g_{2} \leq g_{1},
\end{array}
$$

which proves the "only if" part. The "if" part is proved by observing that the same chain holds in the other direction.

Observe that this ordering is the same as for the UPRA under the uniform power allocation. Thus, we conclude that, for $m=2$, power allocation does not affect the ordering, only the rate allocation does.

This can be further extended to the $m>2$ case as follows.

Proposition 6: A detection ordering $\pi^{*}=\left\{k_{1}, k_{2}, \ldots, k_{m}\right\}$ for the coded V-BLAST under the IPA (and uniform rate allocation) with any number of Tx and Rx antennas is optimum only if

$$
\left|\mathbf{h}_{k_{i-1} \perp}\right| \geq\left|\mathbf{h}_{k_{i} \perp}\right|
$$

for any $\mathbf{h}_{k_{i-1} \perp}$ and $\mathbf{h}_{k_{i} \perp}$ that are non-orthogonal to each other (both are orthogonal to $\left\{\mathbf{h}_{k_{i+1}}, \ldots \mathbf{h}_{k_{m}}\right\}$ ), and any ordering among them offers the same capacity otherwise.

Proof: This is similar to the proof of Proposition 2. Let $\pi^{*}=\left\{k_{1}, . . k_{i-1}, k_{i}, \ldots k_{m}\right\}$ be an optimal ordering and let $\pi=\left\{k_{1}, . . k_{i}, k_{i-1}, \ldots k_{m}\right\}$, i.e. streams $k_{i-1}$ and $k$ are swapped over. Observe that the swapping does not affect the gains of the other streams, i.e. $g_{j}\left(\pi^{*}\right)=g_{j}(\pi) \forall j \neq i-1, i$, where $g_{i}\left(\pi^{*}\right)=\left|\mathbf{h}_{k_{i} \perp}\right|^{2}$ is $i$-th stream (projected) gain under ordering $\pi^{*}$ and $g_{i}(\pi)$ is defined likewise. Now observe that $C\left(\pi^{*}\right) \geq C(\pi)$ only if

$$
\frac{1}{g_{i-1}\left(\pi^{*}\right)}+\frac{1}{g_{i}\left(\pi^{*}\right)} \leq \frac{1}{g_{i-1}(\pi)}+\frac{1}{g_{i}(\pi)},
$$

which implies, from Proposition 5 applied to these two streams, that $\left|\mathbf{h}_{k_{i-1} \perp}\right| \geq\left|\mathbf{h}_{k_{i} \perp}\right|$.

Thus, Foschini ordering (strongest stream detected first) satisfies these necessary pair-wise optimality conditions.

\section{Optimum Ordering Under the IPRA}

It was demonstrated in [19] that, for a given ordering, the well-known water-filling (WF) algorithm does not maximize (in general) the system capacity of the coded V-BLAST via optimum power/rate allocation (IPRA) ${ }^{9}$ and a new algorithm was proposed, the fractional water-filling (FWF), which does so by using the WF on all possible sub-sets of active streams ${ }^{10}$. Since both algorithms provide equal system capacity under an optimal ordering ${ }^{11}$, we consider the WF in this section with understanding that the same results apply to the FWF. The optimal ordering procedure can be formulated as follows:

$$
\pi^{*}=\arg \max _{\pi} \sum_{i} \ln \left(1+\alpha_{i}^{*}(\pi)\left|\mathbf{h}_{k_{i} \perp}\right|^{2} \gamma\right),
$$

where the optimum power allocation $\alpha_{i}^{*}(\pi)$ is given by the WF algorithm,

$$
\alpha_{i}^{*}(\pi)=\left[\mu(\pi)-\frac{1}{\gamma\left|\mathbf{h}_{k_{i} \perp}\right|^{2}}\right]_{+},
$$

where $[x]_{+}=\max \{x, 0\}, \mu(\pi)$ is the water level for a given order $\pi$ and is calculated from the total power constraint. In the general case (any $m$ ), the problem is difficult due to the fact that different ordering may result in different number of active streams. However, if $m=2$, either one or two streams are active and the analysis becomes feasible. The optimal ordering can be characterized as follows.

Proposition 7: The optimum detection ordering for the coded V-BLAST with two Tx and $n \mathrm{Rx}$ antennas under the

\footnotetext{
${ }^{9}$ due to the successive interference cancellation, channel gains become functions of allocated powers, albeit in a binary way: if some streams are allocated zero power, there is no need to project out the interference they create to preceding streams. This dependence is not accounted for in the standard WF algorithm, which assumes that the stream gains are fixed. See [19] for more details.

${ }^{10}$ So that a binary dependence of stream gains on allocated powers is taken into account.

${ }^{11}$ This follows from the fact that, searching among all possible orderings, one always finds an ordering where all the inactive streams of the optimal FWF ordering/allocation are located first; for this ordering, the WF also allocates zero power to those inactive streams so that the WF and FWF are identical for this particular ordering.
} 
IPRA (via the WF or FWF) is to detect the strongest stream last,

$$
\begin{aligned}
\pi^{*} & =\arg \max _{\pi} \sum_{i=1}^{2} \ln \left(1+\alpha_{i}^{*}(\pi)\left|\mathbf{h}_{k_{i} \perp}\right|^{2} \gamma\right) \\
& =\{1,2\} \text { iff }\left|\mathbf{h}_{1}\right| \leq\left|\mathbf{h}_{2}\right|
\end{aligned}
$$

The "only if" part in (19) holds when $\mathbf{h}_{1}$ and $\mathbf{h}_{2}$ are not orthogonal, $\phi \neq \pi / 2$. If $\phi=\pi / 2$ and/or $\left|\mathbf{h}_{1}\right|=\left|\mathbf{h}_{2}\right|$, any ordering delivers the same system capacity.

Proof: See Appendix.

It is a remarkable fact that, whether uniform or optimal power allocation is used, optimal rate allocation always results in the greedy ordering as in (7), (19). This re-enforces our earlier conclusion that it is the rate allocation that is critical for optimal ordering, with power allocation playing no significant role. This conclusion is especially important for the MAC channel, where different users are likely to have different rates.

We are now in a position to establish necessary optimality conditions for $m>2$.

Proposition 8: Consider the ZF V-BLAST under the IPRA with any number of Tx and Rx antennas and any SNR. An optimal ordering satisfies the same necessary conditions as in Proposition 2.

Proof: The key idea of the proof follows that of Proposition 2. Let $\pi^{*}=\left\{k_{1} \ldots k_{m}\right\}$ be optimal ordering. Fix the ordering and power allocation among all streams but $k_{i-1}, k_{i}$. Observe that swapping these two streams and re-allocating power among them (but not the rest) does not affect the capacities of all other streams. Now, apply Proposition 7 to conclude that $\pi^{*}$ is optimal only if $\left|\mathbf{h}_{k_{i-1} \perp}\right| \leq\left|\mathbf{h}_{k_{i} \perp}\right|$.

We observe that adding the optimum power allocation on top of the IRA does not affect the necessary optimality conditions of ordering for any $m$. Unlike the $m=2$ case, channel-only ordering cannot be optimal for $m>2$ since it is SNR-dependent (as numerical experiments show).

\section{SNR GAIN OF ORDERING}

To quantify the impact of optimal ordering, we introduce an SNR gain of ordering, which compares the optimally-ordered and unordered systems. The SNR gain $G$ of ordering is defined as the difference in SNR required by the unordered V-BLAST to achieve the same capacity as the optimally ordered i.e.

$$
C_{\pi *}(\gamma)=C(G \gamma)
$$

where $C_{\pi *}(\gamma)$ and $C(G \gamma)$ are the system capacities with and without optimal ordering.

First, we consider the case of two Tx antennas and the IPRA via the WF, and extend (via bounds) the closed-form results to more general scenarios afterwards. Based on the number of active streams for the $m=2$ case, we consider below three different SNR regimes. This exploits the well-known property of the WF algorithm: while all streams are active at high SNR, only one is active at low SNR.

- Low SNR regime: Both orderings have one active stream:

$$
\gamma \leq \frac{1}{2}\left|\frac{1}{g_{1}}-\frac{1}{g_{2} \beta}\right|,
$$

where $g_{i}=\left|\mathbf{h}_{i}\right|^{2}$ and $\sin ^{2} \phi=\beta$, and we assume, without loss of generality and following (19), that $g_{1} \leq g_{2}$.

- High SNR regime: Both orderings have two active streams:

$$
\gamma>\frac{1}{2}\left(\frac{1}{g_{1} \beta}-\frac{1}{g_{2}}\right) .
$$

- Intermediate SNR regime: The optimum ordering has one active stream and the suboptimum one has two active streams when the SNR is between the bounds in (21) and (22).

Since it is not possible for the sub-optimal ordering to have one active stream and, at the same time, for the optimal one to have two active streams, the characterization above is complete.

Proposition 9: Consider the ZF V-BLAST under the IPRA (via the WF). Assuming unfavorable standard ordering, the SNR gain of ordering in the low SNR regime (as in (21)) is given by

$$
G=\min \left[\frac{1}{\beta}, \frac{g_{2}}{g_{1}}\right],
$$

at high SNR (as in (22)) by

$$
G=1+\frac{1}{2 \gamma}\left(\frac{1}{\beta}-1\right)\left(\frac{1}{g_{1}}-\frac{1}{g_{2}}\right),
$$

and at intermediate SNR by

$$
G=\frac{1}{\gamma}\left(\sqrt{\frac{1+2 g_{2} \gamma}{g_{1} g_{2} \beta}}-\frac{g_{2} \beta+g_{1}}{2 g_{1} g_{2} \beta}\right) .
$$

Proof: Follows from the definition (20) after some manipulations and using the optimal ordering in (19). See Appendix for details.

The SNR gain of ordering is illustrated in Fig. 1, 2. Some conclusions follow from Proposition 9:

* If $g_{1}=g_{2}$ (the per-stream SNRs are equal) and/or $\beta=1$ ( $\mathbf{h}_{1}$ and $\mathbf{h}_{2}$ are orthogonal), there is no gain (both orderings offer the same capacity) at any SNR.

* In the low SNR regime, the gain is SNR-independent, and it is an increasing function of $g_{2} / g_{1}$ and decreasing in $\beta$.

* In the high SNR regime and for fixed $g_{1}, g_{2}$ and $\beta, G$ is decreasing in SNR and $G \rightarrow 1$ as $\gamma \rightarrow \infty$. For fixed $g_{1}$, $g_{2}$ and $\gamma$, it is decreasing in $\beta$. For fixed $g_{2}, \beta$ and $\gamma$, it is decreasing in $g_{1}$.

Below, we obtain a more general result.

Proposition 10: The SNR gain $G$ of ordering of the $m=2$ ZF V-BLAST under any power/rate allocation policy can be bounded as follows:

$$
1 \leq G \leq \min \left[\frac{1}{\beta}, \frac{g_{2}}{g_{1}}\right] .
$$

Proof: Let $C\left(g_{1} \beta, g_{2}, \gamma\right)$ be the system capacity as a function of 1 st and 2 nd stream gains and the SNR. First, we note that $C$ is monotonically increasing in all its arguments and is symmetric in first two under any power/rate allocation (this can be easily verified). To be specific, we consider the IPRA via the WF (the same argument applies to all other policies after a slight change in notations),

$$
C\left(x_{1}, x_{2}, \gamma\right)=\max _{\left\{\alpha_{i}\right\}}\left\{\ln \left(1+\gamma \alpha_{1} x_{1}\right)+\ln \left(1+\gamma \alpha_{2} x_{2}\right)\right\} .
$$




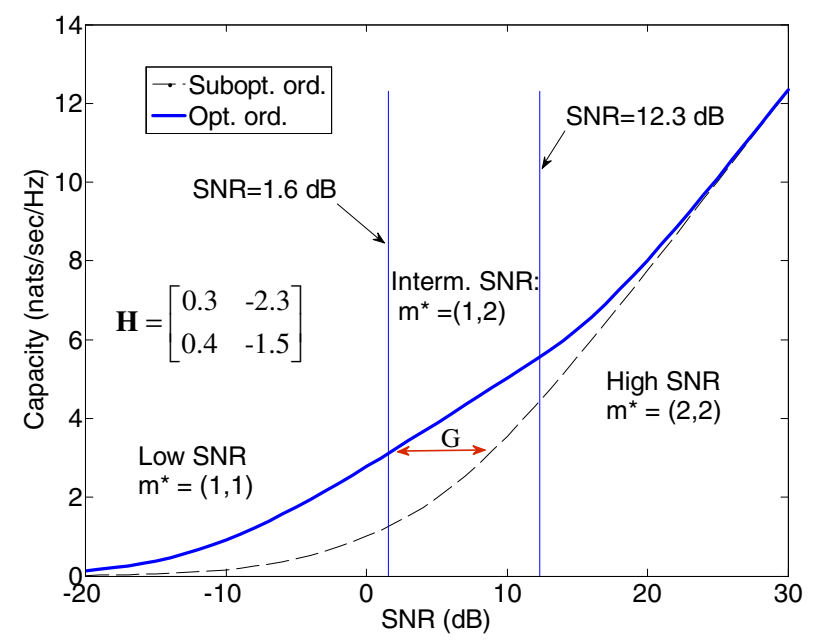

Fig. 1. Impact of ordering on the capacity and the SNR gain $G$ of ordering for the $2 \times 2$ system and given H. $m^{*}$ is the number of active streams for both orderings.

Due to monotonicity of $C\left(x_{1}, x_{2}, \gamma\right)$ in all arguments,

$$
\begin{aligned}
C\left(\beta g_{1}, g_{2}, \gamma\right) & \leq C\left(\beta g_{2}^{2} / g_{1}, g_{2}, \gamma\right) \\
& =C\left(\beta g_{2}, g_{1}, \gamma g_{2} / g_{1}\right) .
\end{aligned}
$$

from which it follows that $G \leq g_{2} / g_{1}$. To prove $G \leq 1 / \beta$, observe that

$$
\begin{aligned}
C\left(\beta g_{1}, g_{2}, \gamma\right) & \leq C\left(g_{1} / \beta, g_{2}, \gamma\right) \\
& =C\left(g_{2}, g_{1} / \beta, \gamma\right) \\
& =C\left(\beta g_{2}, g_{1}, \gamma / \beta\right),
\end{aligned}
$$

where 1 st equality makes use of the symmetry property $C\left(\beta g_{1}, g_{2}, \gamma\right)=C\left(g_{2}, \beta g_{1}, \gamma\right)$.

Thus, there is no gain if $\beta=1$ or $g_{1}=g_{2}$ and little gain if $\beta$ or $g_{2} / g_{1}$ are close to 1 .

Numerical simulations have been carried out to validate the closed-form expressions for the SNR gain of ordering at each SNR regime. Fig. 1 shows the system capacity vs. SNR for the $2 \times 2$ coded V-BLAST system under the IPRA (via WF) for a fixed channel realization $\mathbf{H}$. Note that the SNR gain is a decreasing function of the SNR, as Fig. 2 confirms, so that there is no much advantage from the optimal ordering at high SNR.

Fig. 2 shows the SNR gain of ordering (numerical and analytical) as a function of SNR under the setting in Fig. 1. Note that, in the low SNR regime, the SNR gain is highest and is SNR-independent when both ordering employ only 1 active stream. In the intermediate SNR regime, the gain decreases with the SNR but is still considerable, while it becomes low at high SNR. Thus, we conclude that the major advantage of the optimal ordering is at low SNR, i.e. precisely when it is needed.

The SNR gain of ordering for the general case (any $m$ ) can be bounded as follows.

Proposition 11: Consider the ZF V-BLAST under the IPRA (via the WF or the FWF) for any $m$. Its SNR gain of ordering is bounded at low SNR as follows,

$$
1 \leq G \leq \frac{\left|\mathbf{h}_{\max }\right|^{2}}{\left|\mathbf{h}_{\min }\right|^{2}}
$$

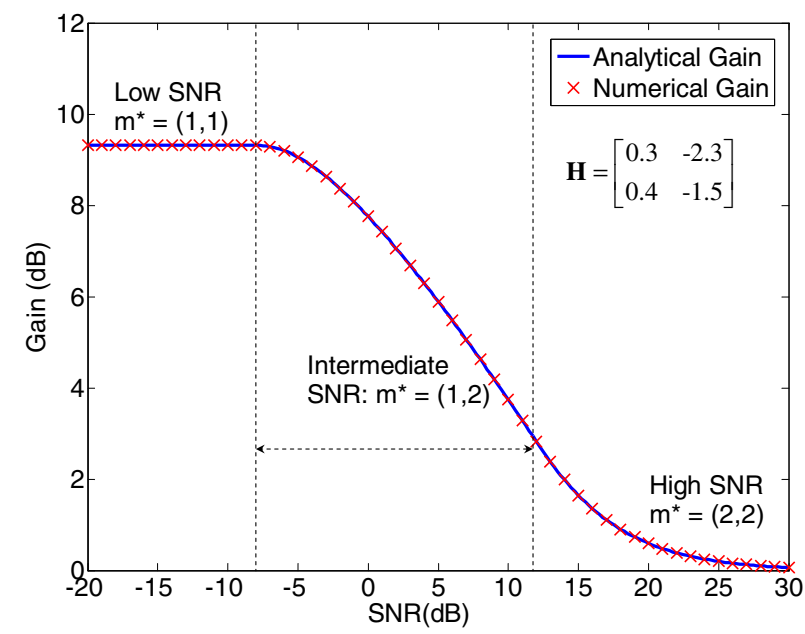

Fig. 2. The SNR gain of ordering vs. SNR (numerical and analytical). The low and intermediate SNR regimes are the largest beneficiaries.

where $\left|\mathbf{h}_{\text {max }}\right|,\left|\mathbf{h}_{\text {min }}\right|$ are the columns with the largest and smallest norms respectively.

Proof: It is a well-known property of the WF (and also the FWF) that only one (strongest) stream is active at low SNR [19]. Therefore, the system capacity under the WF and any ordering $\pi=\left\{k_{1} \ldots k_{m}\right\}$ is

$$
C=\ln \left(1+m \gamma \max _{i}\left|\mathbf{h}_{k_{i} \perp}\right|^{2}\right),
$$

and the best stream gain $\max _{i}\left|\mathbf{h}_{k_{i} \perp}\right|^{2}$ can be bounded as follows:

$$
\left|\mathbf{h}_{\min }\right|^{2} \leq\left|\mathbf{h}_{k_{m}}\right|^{2} \leq \max _{i}\left|\mathbf{h}_{k_{i} \perp}\right|^{2} \leq \max _{i}\left|\mathbf{h}_{k_{i}}\right|^{2}=\left|\mathbf{h}_{\max }\right|^{2},
$$

so that the system capacity is bounded, for any ordering, as

$$
\ln \left(1+m \gamma\left|\mathbf{h}_{\min }\right|^{2}\right) \leq C \leq \ln \left(1+m \gamma\left|\mathbf{h}_{\max }\right|^{2}\right),
$$

from which (30) follows since the lower and upper bounds are ordering-independent. Since only one stream is active, the same argument holds for the FWF. It can be seen (via examples) that the bounds are tight (i.e. achievable, see below).

Note that the lower bound in (30) also holds for any system and any SNR (optimal ordering cannot reduce the capacity), and the upper bound holds at any SNR for a rank-one channel (since only one stream is active in such a channel as the projections result in zero per-stream gain for all streams but the last one). The upper bound is attained in a rank-one channel at any SNR when $\left|\mathbf{h}_{m}\right|=\left|\mathbf{h}_{\min }\right|$ in the unordered system.

Using the results of Section VI, the lower bound in (30) is attained (i.e the ordering does not offer any gain) for the MMSE V-BLAST (under the IRA or the IPRA), in any channel and at any SNR.

For the ZF V-BLAST, it is attained at any SNR and any $m$ when the channel is orthogonal (i.e. all column vectors are orthogonal to each other), since any ordering results in the same capacity as projections are not required. It is also attained for any full-rank channel at high SNR, as the following proposition shows. 
Proposition 12: Consider the ZF V-BLAST under the IRA or the IPRA (via the WF) in any full-rank channel, or under the IPRA via the FWF in any channel, all for any $m$ at high SNR. Its SNR gain of ordering is,

$$
G \rightarrow 1 \text { as } \gamma \rightarrow \infty,
$$

i.e. all orderings deliver asymptotically the same capacity.

Proof: Assume first that the channel is of full rank. Under the IRA, $\alpha_{i}=1$. Under the IPRA, it is well-known that the optimal power allocation is uniform as $\gamma \rightarrow \infty, \alpha_{i} \rightarrow 1$ [19], so that the system capacity, for any ordering $\pi$, is

$$
\begin{aligned}
C\left(\mathbf{H}_{\pi}\right) & =\sum_{i} \ln \left(\gamma\left|\mathbf{h}_{k_{i} \perp}\right|^{2}\right)+o(1) \\
& =m \ln \gamma+\ln \left|\mathbf{H}_{\pi}^{+} \mathbf{H}_{\pi}\right|+o(1) \\
& =m \ln \gamma+2 \sum_{i} \ln \sigma_{i}\left(\mathbf{H}_{\pi}\right)+o(1) \\
& =m \ln \gamma+2 \sum_{i} \ln \sigma_{i}(\mathbf{H})+o(1) \\
& =C(\mathbf{H})+o(1),
\end{aligned}
$$

i.e. unaffected by ordering asymptotically, where we have used the singular value invariance under ordering from Lemma 1 and the property of Gramian $\prod_{i}\left|\mathbf{h}_{k_{i} \perp}\right|^{2}=\left|\mathbf{H}_{\pi}^{+} \mathbf{H}_{\pi}\right|=$ $\prod_{i} \sigma_{i}^{2}\left(\mathbf{H}_{\pi}\right)$ [23]. If channel is rank-deficient, the power is distributed only over a sub-set of streams which correspond to non-zero singular values and the proof goes through when applied to that sub-set.

\section{GREedy ORdERING}

Since an optimal ordering can be computationally demanding for $m>2$, the greedy ordering was introduced in [16][17] as a sub-optimal solution to the optimal ordering problem. Its advantage was demonstrated in [17] via simulations and in [16] via the DMT analysis. Since the latter holds only asymptotically $(S N R \rightarrow \infty)$ and in i.i.d. Rayleigh-fading channel, it is not clear what the finite-SNR implications are $\mathrm{r}^{12}$ and whether this still holds under other fading distributions or for a given, fixed channel (or a given realization of a fading channel). To answer these questions, we will apply the sufficient optimality conditions of Propositions 1 and 7 in this Section.

The greedy ordering algorithm is as follows:

1) Select the largest $\left|\mathbf{h}_{i}\right|$; the corresponding stream is detected last: $k_{m}=\arg \max _{i}\left|\mathbf{h}_{i}\right|$.

2) Select the second largest $\left|\mathbf{h}_{i \perp k_{m}}\right|$; the corresponding stream is detected second last: $k_{m-1}=$ $\arg \max _{i}\left|\mathbf{h}_{i \perp k_{m}}\right|$.

3) Repeat step 2 until finished (always projecting orthogonally to already selected streams). The greedy ordering is $\pi=\left\{k_{1} \ldots k_{m}\right\}$.

i.e. it follows the principle "strongest goes last", unlike the original Foschini ordering [3], which follows the principle "strongest goes first".

We note that its computational complexity is significantly reduced compared to the optimal ordering: while the latter

\footnotetext{
${ }^{12}$ since better DMT does not imply better finite-SNR performance, even at high SNR, see e.g. [24].
}

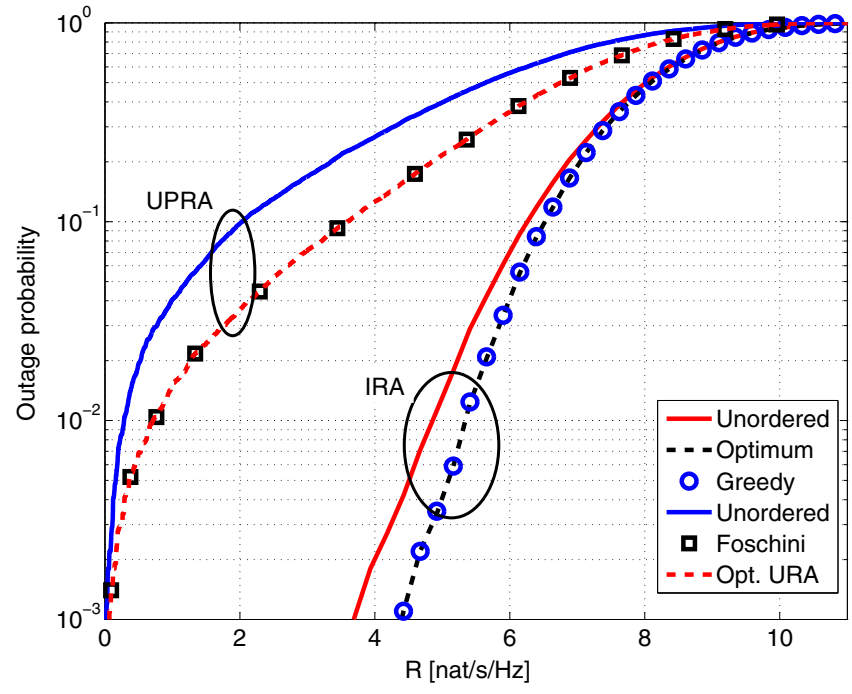

Fig. 3. Empirical outage probability of the $3 \times 3$ unoptimized (UPRA) system and under the IRA, with optimal and sub-optimal orderings; SNR=10dB; $10^{4}$ channel realizations of Rayleigh-fading channel.

compares all $m$ ! possible orderings, the former compares only $m(m+1) / 2-1$ orderings, most of which are in sub-spaces of reduced dimension $(<m)$, i.e. a significant advantage for large $m$.

By noting that this ordering algorithm always satisfies the conditions of Propositions 1 and 7, it follows that the greedy ordering is optimal for any fixed channel (or a given realization of a fading channel, i.e. "point-wise") and at any SNR for the $m=2$ ZF V-BLAST under the IRA or IPRA. This point-wise optimality implies the statistical optimality (in terms of ergodic or outage capacity or outage probability) for any fading distribution and at any SNR. Furthermore, from Propositions 12 and 11 , the greedy ordering is optimal at high and low SNR regimes and for any $m, n$ since it attains the upper bound in (30) when $\left|\mathbf{h}_{m}\right|=\left|\mathbf{h}_{\min }\right|$ under the standard ordering. From Propositions 13, 14, it is optimal for the MMSE V-BLAST.

For larger systems $(m>2)$, this ordering satisfies the necessary optimality conditions in Propositions 2 and 6. This, however, does not guarantee its optimality in general (any SNR, any channel). Indeed, since numerical experiments indicate that an optimal ordering for the $m>2$ case is a function of SNR and since the greedy ordering is not, it cannot be optimal "point-wise" in general. However, extensive numerical experiments show that it is nearly optimal statistically under the IRA or the IPRA in i.i.d. Rayleigh-fading channel, as Fig.3-6 illustrate. The analysis above indicates that the gain of optimal ordering depends significantly on the channel matrix. However, since we wish to have more or less general performance indicator, let us consider below i.i.d. Rayleigh fading and use the system outage probability as its performance measure ${ }^{13}$, which takes into account many channel realizations, not just a few particular channel matrices, so that the aggregate benefit of ordering will be clear.

Fig. 3 compares various ordering strategies in terms of

${ }^{13}$ Since it is the main performance indicator in quasy-static fading channels [21]. This is also inline with the existing literature, see e.g. [5]-[10][19]. 


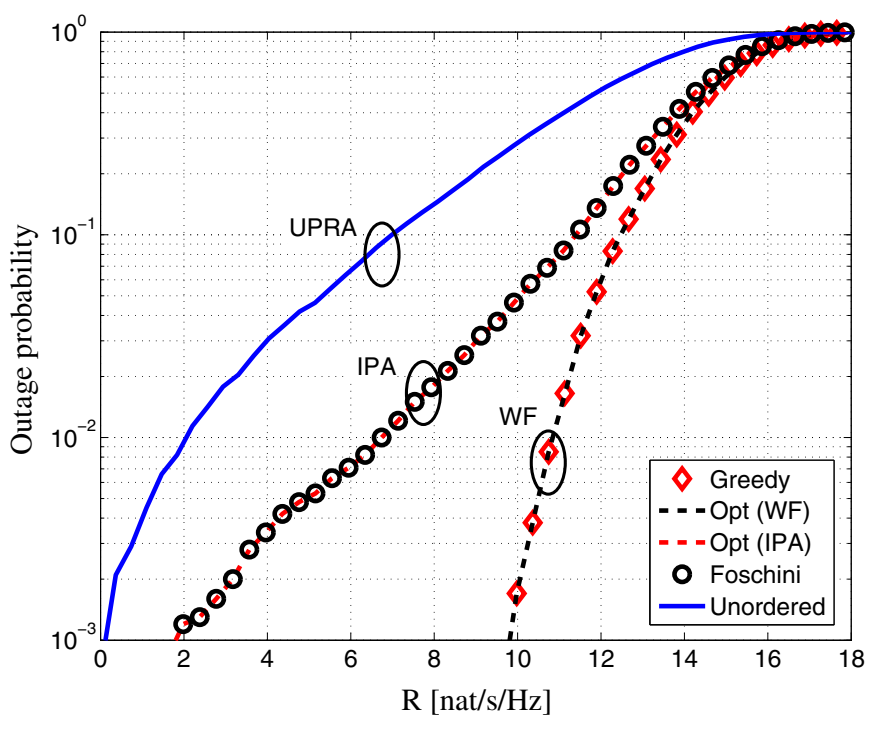

Fig. 4. Empirical outage probability of the $3 \times 3$ unoptimized (UPRA), unordered system compared to the IPA under Foschini and optimal orderings, and the WF under the greedy and optimum orderings; SNR=20dB; $10^{4}$ channel realizations.

the outage probability in i.i.d. Rayleigh-fading channel. The optimality of Foschini ordering under the UPRA is clearly observed. It can also be seen that the greedy ordering under the IRA is almost optimum. Finally, the significantly-better performance of the ordered rate-optimized system as compared to the unordered, unoptimized one is evident: while $P_{\text {out }}=10^{-3}$ is essentially unachievable by the latter, it is achieved by the former at $R \approx 4.5[\mathrm{nat} / \mathrm{s} / \mathrm{Hz}]$.

The performance of the greedy and Foschini orderings are evaluated under the WF (IPRA) and the IPA respectively in Fig. 4. It can be seen that both orderings are almost optimum for each respective case and perform significantly better than the unordered, unoptimized system. Specifically, while $P_{\text {out }}=$ $10^{-3}$ is achieved at $R \approx 0.1[\mathrm{nat} / \mathrm{s} / \mathrm{Hz}]$ by the unoptimized, unordered system, $R \approx 2$ and $10[\mathrm{nat} / \mathrm{s} / \mathrm{Hz}]$ are delivered by the IPA and IPRA respectively under the greedy ordering.

The near-optimality of the greedy ordering is also observed for larger systems under the IPRA and at different SNR regimes, as Fig. 5 and 6 demonstrate. We attribute this to the fact that the greedy ordering, while not being optimal in general, does rule out many "bad" orderings so that the remaining ordering does perform well. Given that the greedy ordering has much smaller computational complexity compared to the exhaustive search of an optimal ordering, the former is a valuable practical alternative.

\section{MMSE V-BLAST}

In this section, we consider the MMSE V-BLAST, where nulling the interference from yet-to-be-detected symbols is balanced against the noise enhancement so that per-stream SNR is maximized [21]. We demonstrate that, contrary to the ZF V-BLAST considered before, any ordering is optimal (delivers the same capacity) when the IRA or the IPRA are used.

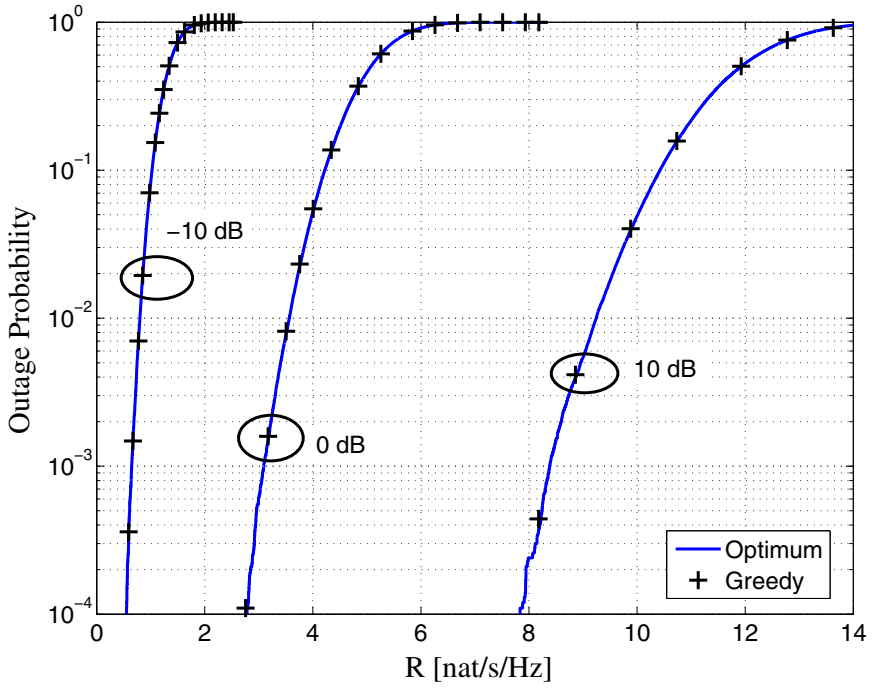

Fig. 5. Empirical outage probability of the greedy and optimum orderings under WF (IPRA) for the $4 \times 4$ system in i.i.d. Rayleigh fading channel via MC with $10^{5}$ channel realizations; SNR $=-10,0,10 \mathrm{~dB}$.

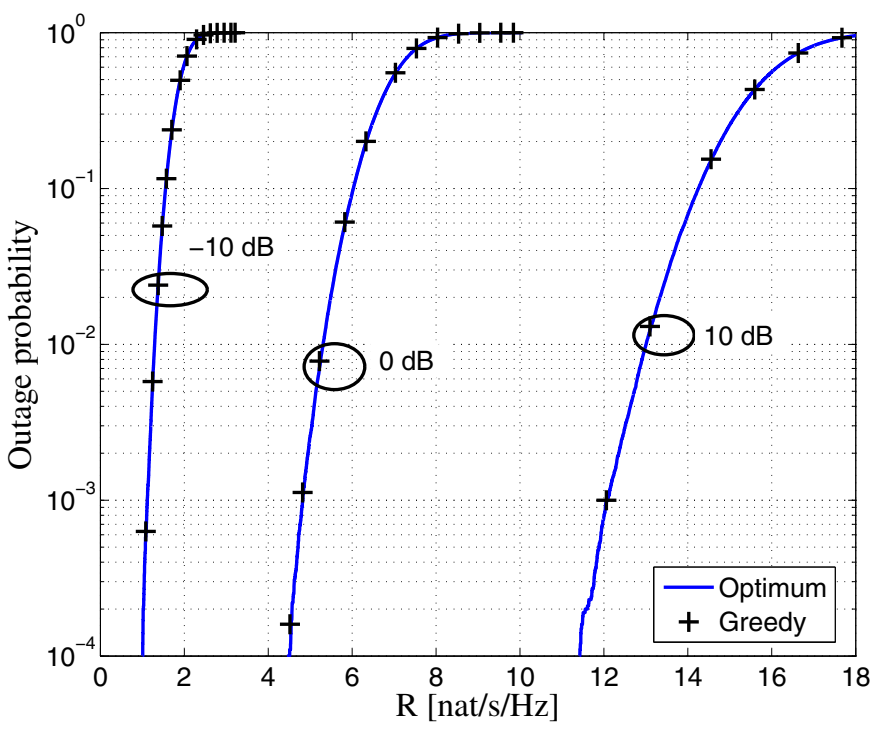

Fig. 6. Empirical outage probability of the greedy and optimum orderings under WF (IPRA) for the $5 \times 5$ system in i.i.d. Rayleigh fading channel via MC with $10^{5}$ channel realizations; SNR $=-10,0,10 \mathrm{~dB}$.

\section{A. MMSE V-BLAST under IRA}

Under the MMSE combining at each step, the per-stream SNR at step $i$ under the standard ordering is [21]

$$
\gamma_{i}=\mathbf{h}_{i}^{+}\left(\sigma_{0}^{2} \mathbf{I}+\mathbf{H}_{i} \mathbf{H}_{i}^{+}\right)^{-1} \mathbf{h}_{i},
$$

where $\mathbf{H}_{i}=\left[\mathbf{h}_{i+1} \ldots \mathbf{h}_{m}\right]$ is the reduced channel matrix representing yet-to-be detected streams $(i+1) \ldots m$, and the stream capacity is $\ln \left(1+\gamma_{i}\right)$ so that the total system capacity is

$$
C_{I R A}=\sum_{i} \ln \left(1+\gamma_{i}\right) .
$$

It follows that, in this system, any ordering delivers the same capacity.

Proposition 13: Consider the MMSE V-BLAST under the IRA (and the uniform power allocation among the streams). 
Then, any ordering is optimal, i.e. delivers the same system capacity,

$$
C_{I R A}=C_{I R A}\left(\mathbf{H}_{\pi}\right) \forall \pi
$$

at any SNR.

Proof: See Appendix.

It is a remarkable fact that any ordering works equally well with coded MMSE V-BLAST under the IRA, since it eliminates the need for computationally-intensive ordering procedure. This provides an extra incentive for using MMSE rather than ZF interference nulling, beyond the well-known better uncoded error rate performance of the former. This conclusion is also in stark contrast to the case of coded MMSE V-BLAST under the UPRA, where optimal ordering does provide significant performance improvement [10].

We further proceed to establish this property for the MMSE V-BLAST under the IPRA (via the WF or FWF) as well.

\section{B. MMSE V-BLAST under IPRA}

Under the IPRA (via the WF or FWF), per-stream powers are optimally allocated so that the channel model in (1) applies and the total system capacity is

$$
C_{I P R A}=\sum_{i} \ln \left(1+\widetilde{\gamma}_{i}\right),
$$

where the per-stream SNR $\widetilde{\gamma}_{i}$ is

$$
\widetilde{\gamma}_{i}=\widetilde{\mathbf{h}}_{i}^{+}\left(\sigma_{0}^{2} \mathbf{I}+\widetilde{\mathbf{H}}_{i} \widetilde{\mathbf{H}}_{i}^{+}\right)^{-1} \widetilde{\mathbf{h}}_{i},
$$

where $\widetilde{\mathbf{H}}=\mathbf{H} \Lambda$ is the equivalent channel matrix (which accounts for non-uniform power allocation) and $\widetilde{\mathbf{H}}_{i}, \widetilde{\mathbf{h}}_{i}$ are defined likewise.

Proposition 14: Consider the MMSE V-BLAST under the IPRA (via the WF or FWF). Then, any ordering delivers the same system capacity (i.e. optimal),

$$
C_{I P R A}=C_{I P R A}\left(\mathbf{H}_{\pi}\right) \forall \pi,
$$

at any SNR.

Proof: Follows from Proposition 13 via the substitution $\mathbf{H} \rightarrow \widetilde{\mathbf{H}}$, so that for any power allocation, including the optimal one, any channel ordering delivers the same system capacity.

\section{CONCLUSION}

An optimal ordering problem for the coded ZF and MMSE V-BLAST has been considered in this paper. The sufficient and necessary optimality conditions have been established under four different power/rate allocation policies (UPRA, IPA, IRA and IPRA), motivated by modern adaptive systems. In the case of $m=2 \mathrm{ZF} \mathrm{V-BLAST}$, the optimal ordering is shown to be the greedy ordering ("strongest goes last") under the IRA/IPRA at any SNR and for any fixed channel, while the Foschini ordering ("strongest goes first") is optimal under the UPRA/IPA, i.e. it is the rate allocation policy that has a major impact on the optimal ordering procedure. The point-wise optimality of the greedy ordering translates into its statistical optimality (in terms of ergodic or outage capacity or outage probability) under any fading and at any SNR. The SNR gain of ordering was introduced and studied to quantify the beneficial impact of ordering. Any ordering is shown to be optimal for the coded MMSE V-BLAST under the IRA/IPRA.

\section{APPENDIX}

\section{A. Proof of Proposition 7}

First, observe that any ordering is optimal when $g_{1}=g_{2}$ so we further assume that $g_{2}>g_{1}$. The key idea of the proof is to demonstrate that $\pi=\{2,1\}$ cannot be optimal at any SNR. The main difficulty is that two orderings may have different number of active streams under the WF (or FWF), which makes the algebra very lengthy (except when only 1 stream is active in both cases). Instead, we demonstrate that swapping the streams without changing the power allocation provides higher capacity. Let $C_{1}=C(\pi)$ be the capacity of ordering $\pi$,

$$
C_{1}=\ln \left(1+\gamma \alpha_{1} \beta g_{2}\right)+\ln \left(1+\gamma \alpha_{2} g_{1}\right),
$$

and $C_{2}$ be the capacity of ordering $\pi^{*}=\{1,2\}$ with the same power allocation as that of $\pi$,

$$
C_{2}=\ln \left(1+\gamma \alpha_{1} \beta g_{1}\right)+\ln \left(1+\gamma \alpha_{2} g_{2}\right) .
$$

Assume first that $\alpha_{2}>\alpha_{1}$ and observe that

$$
\begin{aligned}
e^{C_{2}}-e^{C_{1}}= & \left(1+\gamma \alpha_{1} \beta g_{1}\right)\left(1+\gamma \alpha_{2} g_{2}\right) \\
& -\left(1+\gamma \alpha_{1} \beta g_{2}\right)\left(1+\gamma \alpha_{2} g_{1}\right) \\
= & \left(\alpha_{2}-\alpha_{1} \beta\right)\left(g_{2}-g_{1}\right)>0,
\end{aligned}
$$

so that $C_{1}<C_{2} \leq C\left(\pi^{*}\right)$, where the last inequality is due to the fact that $\left\{\alpha_{1}, \alpha_{2}\right\}$ is not optimal under $\pi^{*}$. If $\alpha_{1} \geq \alpha_{2}$, consider $g_{2}^{\prime}=\alpha_{1} g_{2}>g_{1}^{\prime}=\alpha_{2} g_{1}$ as an equivalent channel (with uniform power allocation) and swap the streams so that

$$
C_{1}<\ln \left(1+\gamma \beta g_{1}^{\prime}\right)+\ln \left(1+\gamma g_{2}^{\prime}\right) \leq C\left(\pi^{*}\right),
$$

where 1st inequality is from Proposition 1 ( $\pi$ is not optimal on the equivalent channel $\left\{g_{2}^{\prime}, g_{1}^{\prime}\right\}$ since $\left.g_{2}^{\prime}>g_{1}^{\prime}\right)$, and 2 nd one from the fact that $\left\{\alpha_{1}, \alpha_{2}\right\}$ is not optimal under $\pi^{*}$. Thus, in both cases, $C_{1} \leq C\left(\pi^{*}\right)$, which proves the "if" part. The "only if" part follows from essentially the same argument.

\section{B. Proof of Proposition 9}

First, we demonstrate that the SNR regimes in (21) and (22) indeed correspond to 1 and 2 active streams respectively for both orderings.

Let $\pi^{*}=\{1,2\}$ and $\pi=\{2,1\}$. Then, from (18), there is only one active stream for $\pi^{*}$ if $\mu\left(\pi^{*}\right) \leq 1 /\left(g_{1} \beta \gamma\right)$ (the weakest stream is inactive), where

$$
\mu\left(\pi^{*}\right)=1+\frac{1}{2 \gamma}\left(\frac{1}{g_{1} \beta}+\frac{1}{g_{2}}\right),
$$

which can be expressed as

$$
\gamma \leq \frac{g_{2}-g_{1} \beta}{2 g_{1} g_{2} \beta} .
$$

Applying the same reasoning to $\pi$, one obtains

$$
\gamma \leq \frac{\left|g_{1}-g_{2} \beta\right|}{2 g_{1} g_{2} \beta} .
$$

Using the fact that $g_{2}-g_{1} \beta \geq\left|g_{1}-g_{2} \beta\right|$ for $g_{2} \geq g_{1}$, the last bound is tighter, which results in (21). This also proves that the scenario where the optimal ordering has 2 active streams but sub-optimal - only one is impossible. Following the same reasoning and reversing the inequalities in (47) and (48), one 
obtains (22). As a side remark, we note that the intermediate SNR regime does not exist if $g_{1}=g_{2}$ or/and $\beta=1$ (any ordering is optimal in this case).

Let $C_{1(2)}$ be the capacity under $\pi\left(\pi^{*}\right)$. To prove (23), consider 1 st the case $g_{1}>g_{2} \beta$ so that $C_{i}=\ln \left(1+2 \gamma g_{i}\right)$ and using this in the gain definition (20) results in $G=g_{2} / g_{1}$. Now let $g_{1}<g_{2} \beta$, so that $C_{2}$ stays the same and $C_{1}=\ln \left(1+2 \gamma \beta g_{2}\right)$ and the comparison reveals $G=1 / \beta$, which proves (23).

To prove (25), notice that $C_{i}=\ln \left(\mu_{i}^{2} \beta g_{1} g_{2} \gamma^{2}\right)$ when both streams are active for both orderings, where $\mu_{i}$ is the corresponding water level: $\mu_{2}=\mu\left(\pi^{*}\right)$ and

$$
\mu_{1}=\mu(\pi)=1+\frac{1}{2 \gamma}\left(\frac{1}{g_{2} \beta}+\frac{1}{g_{1}}\right) .
$$

Using $C_{i}$ in the gain definition results in (25), after some manipulations.

Finally, to prove (24), notice that $C_{1}=\ln \left(1+2 \gamma g_{2}\right)$ and $C_{2}=\ln \left(\mu_{2}^{2} \beta g_{1} g_{2} \gamma^{2}\right)$. Using these in the gain definition, one obtains, after some manipulations, (24).

\section{Proof of Proposition 13}

It is well-known (see e.g. [4][21]) that the MMSE V-BLAST under the IRA achieves the full MIMO channel capacity (under the isotropic signalling), i.e. is information-lossless ${ }^{14}$,

$$
\begin{aligned}
C_{I R A} & =\sum_{i} \ln \left(1+\gamma_{i}\right) \\
& =\ln \left|\mathbf{I}+\gamma \mathbf{H} \mathbf{H}^{+}\right| \\
& =\sum_{i} \ln \left(1+\gamma \sigma_{i}^{2}(\mathbf{H})\right),
\end{aligned}
$$

where $\gamma=1 / \sigma_{0}^{2}$ and $\sigma_{i}(\mathbf{H})$ are singular values of $\mathbf{H}$. Any detection (stream) ordering is equivalent to permutation (reordering) of the columns of $\mathbf{H}$. We further need the following technical lemma.

Lemma 1: Singular values of any matrix are not affected by re-ordering of its columns, i.e.

$$
\sigma_{i}(\mathbf{H})=\sigma_{i}\left(\mathbf{H}_{\pi}\right)
$$

Proof: Consider the singular value decomposition $\mathbf{H}=$ $\mathbf{U} \boldsymbol{\Sigma} \mathbf{V}^{+}$, where $\mathbf{U}, \mathbf{V}$ are unitary matrices of left and right singular vectors of $\mathbf{H}$ and $\boldsymbol{\Sigma}$ is a diagonal matrix of its singular values. Observe that

$$
\begin{aligned}
\mathbf{H}_{\pi} & =\mathbf{H} \mathbf{P}_{\pi} \\
& =\mathbf{U} \boldsymbol{\Sigma} \mathbf{V}^{+} \mathbf{P}_{\pi} \\
& =\mathbf{U} \boldsymbol{\Sigma} \mathbf{V}_{\pi}^{+},
\end{aligned}
$$

where $\mathbf{P}_{\pi}=\left[\mathbf{e}_{k_{1}} \ldots \mathbf{e}_{k_{m}}\right]$ is the permutation matrix corresponding to permutation $\pi=\left\{k_{1} \ldots k_{m}\right\}, \mathbf{e}_{k}=[0 . .0,1,0 . .0]^{T}$ is a standard basis vector with all zero entries except $k$-th one, and $\mathbf{V}_{\pi}^{+}=\mathbf{V}^{+} \mathbf{P}_{\pi}$, where we have used the fact that the column permutation is equivalent to right multiplication by $\mathbf{P}_{\pi}$. It is straightforward to verify that simultaneous permutation of the entries of any two vectors does preserve their scalar product (and thus the Euclidean norm), $\mathbf{a}^{+} \mathbf{b}=\mathbf{a}_{\pi}^{+} \mathbf{b}_{\pi}$, where $\mathbf{a}_{\pi}\left(\mathbf{b}_{\pi}\right)$ has the same entries as $\mathbf{a}(\mathbf{b})$ but arranged according

\footnotetext{
${ }^{14}$ It essentially implements the chain rule of mutual information via the SIC and hence is an information-preserving processing [21] .
}

to ordering $\pi$. Therefore, since $\mathbf{V}^{+}$has orthonormal rows, so is $\mathbf{V}_{\pi}^{+}$and hence it is unitary. Thus, (51) is a valid singular value decomposition of $\mathbf{H}_{\pi}$, which demonstrates that only right singular vectors are affected by the column permutation while the left ones and singular values are not affected. As a side remark, we note that column permutation does affect eigenvalues of a matrix.

Applying this Lemma to (50), one obtains:

$$
\begin{aligned}
C_{I R A}\left(\mathbf{H}_{\pi}\right) & =\sum_{i} \ln \left(1+\gamma \sigma_{i}^{2}\left(\mathbf{H}_{\pi}\right)\right) \\
& =\sum_{i} \ln \left(1+\gamma \sigma_{i}^{2}(\mathbf{H})\right) \\
& =C_{I R A}(\mathbf{H}) .
\end{aligned}
$$

\section{REFERENCES}

[1] E. Biglieri, et al., MIMO Wireless Communications. Cambridge University Press, 2007.

[2] P. W. Wolniansky, G. J. Foschini, G. D. Golden, and R. A. Valenzuela, "'V-BLAST: an architecture for realizing very high data rates over the rich-scattering wireless channel," in Proc. 1998 International Symposium on Signals, Systems, and Electronics, p. 295-300.

[3] G. J. Foschini, et al., "Simplified processing for high spectral efficiency wireless communication employing multi-element arrays," IEEE J. Sel. Areas Commun., vol. 17, no. 11, pp. 1841-1852, Nov. 1999.

[4] G. J. Foschini, et al., "Analysis and performance of some basic spacetime architectures," IEEE J. Sel. Areas Commun., vol. 21, no. 3, pp. 281-320, Apr. 2003.

[5] N. Prasad and M. Varanasi, "Analysis of decision feedback detection for MIMO Rayleigh-fading channels and the optimization of power and rate allocations," IEEE Trans. Inf. Theory, vol. 50, no. 6, pp. 1009-1025, 2004.

[6] S. Loyka and F. Gagnon, "Performance analysis of the V-BLAST algorithm: an analytical approach," IEEE Trans. Wireless Commun., vol.3, no. 4, pp. 1326-1337, Jul. 2004.

[7] J. Choi, "Nulling and cancellation detector for MIMO channels and its application to multistage receiver for coded signals: performance and optimization," IEEE Trans. Wireless Commun., vol. 5, no. 5, pp. 12071216, May 2006.

[8] S. Loyka and F. Gagnon, "V-BLAST without optimal ordering: analytical performance evaluation for Rayleigh fading channels," IEEE Trans. Commun., vol. 54, no. 6, pp. 1109-1120, June 2006.

[9] S. Loyka and F. Gagnon, "On outage and error rate analysis of the ordered V-BLAST," IEEE Trans. Wireless Commun., vol. 7, no. 10, pp. 36793685, Oct. 2008.

[10] Y. Jiang, M. K. Varanasi, and J. Li, "Performance analysis of ZF and MMSE equalizers for MIMO systems: an in-depth study of the high SNR regime," IEEE Trans. Inf. Theory, vol. 57, no. 4, Apr. 2011.

[11] S. Nam, O. Shin, and K. Lee, "Transmit power allocation for a modified V-BLAST system," IEEE Trans. Commun., vol. 52, no. 7, pp. 1074-1079, Jul. 2004.

[12] R. Kalbasi, D. Falconer, and A. Banihashemi, "Optimum power allocation for a V-BLAST system with two antennas at the transmitter," IEEE Commun. Lett., vol. 9, no. 9, pp. 826-828, Sept. 2005.

[13] H. Lee and I. Lee, "New approach to error compensation in coded V-BLAST OFDM systems," IEEE Trans. Commun., vol. 55, no. 2, pp. 345-355, 2007.

[14] V. Kostina and S. Loyka, "On optimum power allocation for the VBLAST," IEEE Trans. Commun., vol. 56, no. 6, pp. 999-1012, June 2008.

[15] L. Barbero and J. Thompson, "Fixing the complexity of the sphere decoder for MIMO detection," IEEE Trans. Wireless Commun., vol. 7, no. 6, pp. 2131-2142, June 2008.

[16] Y. Jiang and M. Varanasi, "Spatial multiplexing architectures with jointly designed rate-tailoring and ordered BLAST decoding-part I: diversitymultiplexing tradeoff analysis," IEEE Trans. Wireless Commun., vol. 7, no. 8, pp. 3252-3261, Aug. 2008.

[17] R. Zhang and J. Cioffi, "Approaching MIMO-OFDM capacity with zeroforcing V-BLAST decoding and optimized power, rate, and antennamapping feedback," IEEE Trans. Signal Process., vol. 56, no. 10, pp. 5191-5203, Oct. 2008. 
[18] V. Kostina and S. Loyka, "Optimum power and rate allocation for coded V-BLAST: average optimization,” IEEE Trans. Commun., vol. 59, no. 3, pp. 877-887, Mar. 2011.

[19] V. Kostina and S. Loyka, "Optimum power and rate allocation for coded V-BLAST: instantaneous optimization," IEEE Trans. Commun., vol. 59, no. 10, pp. 2841-2850, Oct. 2011.

[20] G. Caire and K. Kumar, "Information theoretic foundations of adaptive coded modulation," Proc. IEEE, vol. 95, no. 12, pp. 2274-2298, 2007.

[21] D. Tse and P. Viswanath, Fundamentals of Wireless Communication. Cambridge University Press, 2005.

[22] L. Boyd and S. Vandenberghe, Convex Optimization. Cambridge University Press, 2004

[23] F. R. Gantmacher, The Theory of Matrices, Vol. 1. AMS Chelsea Publishing, 1977.

[24] S. Loyka and G. Levin, "Finite-SNR diversity-multiplexing tradeoff via asymptotic analysis of large MIMO systems," IEEE Trans. Inf. Theory, vol. 56, no. 10, pp. 4781-4792, Oct. 2010.

[25] S. Loyka and G. Levin, "Diversity-multiplexing tradeoff in the low-SNR regime,” IEEE Commun. Lett., vol. 15, no. 5, pp. 542-544, May 2011.

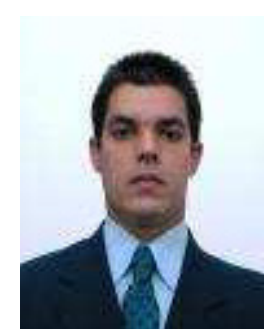

Alain Uriarte Toboso was born in Havana, Cuba. He received the Bachelor's degree in Telecommunications and Electronics Engineering from the Polytechnic Institute Jose Antonio Echeverria (ISPJAE), Havana, Cuba in 2007 and the Master's degree (M.A.Sc.) in Electrical Engineering from the University of Ottawa, Canada, in 2012. His research interests include wireless communications, MIMO systems and smart antennas.

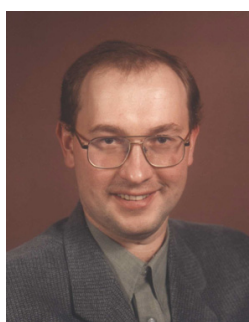

Sergey Loyka was born in Minsk, Belarus. He received the $\mathrm{Ph}$.D. degree in Radio Engineering from the Belorussian State University of Informatics and Radioelectronics (BSUIR), Minsk, Belarus in 1995 and the M.S. degree with honors from Minsk Radioengineering Institute, Minsk, Belarus in 1992. Since 2001 he has been a faculty member at the School of Electrical Engineering and Computer Science, University of Ottawa, Canada. Prior to that, he was a research fellow in the Laboratory of Communications and Integrated Microelectronics (LACIME) of Ecole de Technologie Superieure, Montreal, Canada; a senior scientist at the Electromagnetic Compatibility Laboratory of BSUIR, Belarus; an invited scientist at the Laboratory of Electromagnetism and Acoustic (LEMA), Swiss Federal Institute of Technology, Lausanne, Switzerland. His research areas are wireless communications and networks and, in particular, MIMO systems and security aspects of such systems, in which he has published extensively. He received a number of awards from the URSI, the IEEE, the Swiss, Belarus and former USSR governments, and the Soros Foundation.

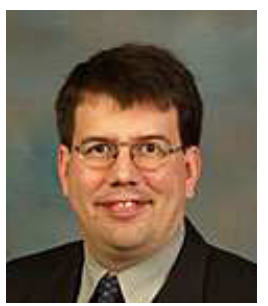

Francois Gagnon (S'87-M'87-SM'99) was born in Quebec City, PQ., Canada. He received the B.Eng. and $\mathrm{Ph} . \mathrm{D}$. degrees in electrical engineering from Ecole Polytechnique de Montreal, Canada. Since 1991, he has been a Professor in the Department of Electrical Engineering, Ecole de technologie superieure, Montreal, Canada. $\mathrm{He}$ has chaired the department from 1999 to 2001 and is now the holder of the Ultra Electronics (TCS) Chair in Wireless Communication in the same university. His research interest covers wireless high speed communications, modulation, coding, high speed DSP implementations and military point to point communications. He has been very involved in the creation of the new generation of High Capacity Line Of Sight military radios offered by the Canadian Marconi Corporation, which is now Ultra Electronics Tactical Communication Systems. The company has received, for this product, a 'Coin of excellence' from the US army for performance and reliability. 